\title{
Early Vascular Response to Ultrathin Biodegradable Polymer Sirolimus-Eluting Stents for the Treatment of ST-Elevation Myocardial Infarction After Plaque Rupture
}

\author{
Yosuke Oishi, ${ }^{1}$ MD, Hiroaki Tsujita, ${ }^{1} \mathrm{MD}$, Kunihiro Ogura, ${ }^{1} \mathrm{MD}$, Naoki Matsukawa, ${ }^{2}$, \\ Hideaki Tanaka, ${ }^{1}$ MD, Ryota Masaki, ${ }^{1}$ MD, Koshiro Sakai, ${ }^{1}$ MD, Teruo Sekimoto, ${ }^{1}$ MD, \\ Seita Kondo, ${ }^{1}$ MD, Shigeto Tsukamoto, ${ }^{1}$ MD, Hidenari Matsumoto, ${ }^{1}$ MD, Hiroyoshi Mori, ${ }^{3}$ MD, \\ Ken Arai, ${ }^{4}$ MD, Kosuke Nomura, ${ }^{5}$ MD, Syunya Sato, ${ }^{6}$ MD, Myong Hwa Yamamoto, ${ }^{6}$ MD, \\ Ryota Kosaki, ${ }^{7}$ MD, Kohei Wakabayashi, ${ }^{7}$ MD, Rikuo Sakai, ${ }^{8}$ MD, Taito Arai, ${ }^{8}$ MD, \\ Hiroshi Suzuki, ${ }^{3}$ MD, Masahiko Ochiai, ${ }^{6}$ MD and Toshiro Shinke, ${ }^{1}$ MD
}

\begin{abstract}
Summary
Recent clinical studies suggest that newer-generation drug-eluting stents that combine ultrathin struts and nanocoating (biodegradable polymer sirolimus-eluting stents, BP-SES) could improve long-term clinical outcomes in patients with ST-elevation myocardial infarction (STEMI) undergoing primary percutaneous coronary intervention (PCI). However, the early vascular response to BP-SES in these patients has not been investigated so far.

We examined this response in 20 patients with STEMI caused by plaque rupture using frequency-domain optical coherence tomography (OCT) to understand the underlying mechanisms. Plaque rupture was diagnosed by OCT before PCI with BP-SES implantation was performed. OCT was again performed before the final angiography (post-PCI) and after 2 weeks (2W-OCT).

BP-SES placement caused protrusion of atherothrombotic material into the stent lumen and incomplete stent apposition in all patients. After 2 weeks, incomplete stent apposition was significantly reduced (\% malapposed struts: post-PCI $4.7 \pm 3.3 \% ; 2 \mathrm{~W}$-OCT $0.9 \pm 1.2 \%$; $P<0.0001$ ), and the percentage of uncovered struts also significantly decreased $(\%$ uncovered struts: post-PCI; $69.8 \pm 18.3 \%$ : 2W-OCT; $29.6 \pm 11.0 \%, P<$ 0.0001 ). The maximum protrusion area of the atherothrombotic burden was significantly reduced (post-PCI 1.36 $\pm 0.70 \mathrm{~mm}^{2} ; 2 \mathrm{~W}$-OCT $\left.0.98 \pm 0.55 \mathrm{~mm}^{2} ; P=0.004\right)$.

This study on the early vascular responses following BP-SES implantation showed rapid resolution of atherothrombotic material and progression of strut apposition and coverage.
\end{abstract}

(UMIN000041324)

(Int Heart J 2021; 62: 42-49)

Key words: Acute coronary syndrome, Drug-eluting stents, Strut thickness, Percutaneous coronary intervention, Optical coherence tomography

$\mathrm{D}$ urable-polymer everolimus-eluting stents (DPEES) have shown a lower incidence of stent thrombosis than bare-metal stents (BMS) in patients with ST-elevation myocardial infarction (STEMI) and the differences were observed within the first 2 weeks after implantation in the EXAMINATION study. ${ }^{1)}$ This favorable effect was partly explained by thin struts and the coating of DP-EES with fluorinated copolymer composed of vinylidene fluoride and hexafluoropropylene monomers, which was shown to exhibit thromboresistance and hemocompatibility. ${ }^{2)}$ Even in the very early phase after implan- tation of DP-EES in patients with STEMI, this antithrombotic potential and thin struts resulted in the progression of strut coverage and a significant reduction in thrombus formation. ${ }^{3}$

Recent clinical studies suggest that a newergeneration drug-eluting stent (DES), combining ultrathin struts and nanocoating with biodegradable polymer sirolimus-eluting stents (BP-SES), showed lower target lesion failure than that of DP-EES.) It was concluded that BP-SES was more beneficial than DP-EES in patients with STEMI. ${ }^{5)}$ Thereafter, Iglesias, et al..$^{6}$ consequently re-

From the ${ }^{1}$ Division of Cardiology, Department of Medicine, Showa University School of Medicine, Tokyo, Japan, ${ }^{2}$ Department of Legal Medicine, Showa University School of Medicine, Tokyo, Japan, ${ }^{3}$ Department of Cardiology, Showa University Fujigaoka Hospital, Yokohama, Japan, ${ }^{4}$ Department of Medicine, Hitachi Medical Center Hospital, Hitachi, Japan, ${ }^{5}$ Department of Cardiology, Kanto Rosai Hospital, Kawasaki, Japan, ${ }^{6}$ Division of Cardiology and Cardiac Catheterization Laboratories, Showa University Northern Yokohama Hospital, Yokohama, Japan, ${ }^{7}$ Cardiovascular Centre, Showa University Koto-Toyosu Hospital, Tokyo, Japan and ${ }^{8}$ Department of Cardiology, Koyama Memorial Hospital, Kashima, Japan.

Address for correspondence: Toshiro Shinke, MD, Division of Cardiology, Department of Medicine, Showa University School of Medicine, 1-5-8 Hatanodai, Shinagawa-ku, Tokyo 142-8666, Japan. E-mail: shinke@med.showa-u.ac.jp

Received for publication June 28, 2020. Revised and accepted September 7, 2020.

doi: $10.1536 /$ ihj.20-458

All rights reserved by the International Heart Journal Association. 
ported that BP-SES achieved target lesion failure rates superior to those of DP-EES in patients with STEMI after 1 year, and the Kaplan-Meier curve of BP-SES after primary PCI was more favorable than that of DP-EES during the very early postintervention phase of 1 month.

The ultrathin struts of BP-SES surrounded nanocoating with poly-l-lactic acid, which shows lower electron transfer out of the stent material than other stent coatings, and also amorphous hydrogenated silicon-carbide coatings may effectively promote early progression of strut coverage and mitigate thrombus formation. ${ }^{7,8)}$ Still, there was no significant difference in antithrombogenicity between BPSES and DP-EES in an ex vivo model. ${ }^{9)}$ It might be possible that BP-SES are suitable for the treatment of thrombotic lesions in STEMI patients. There have been few reports, however, on the vascular response to BP-SES in the very early phase.

Early after stent placement to STEMI patients especially within 2 weeks has been supposed to be an important time window of subacute stent thrombosis. ${ }^{10)}$ Thus, Morino, et al..$^{3)}$ demonstrated the promoted vascular healing at 2 weeks after DP-EES implantation to STEMI patients.

Frequency-domain optical coherence tomography (OCT) with high-resolution imaging is appropriate for examining the vascular response to stents because it enables the evaluation of in-stent structures, strut coverage, strut malapposition, and thrombus volume. ${ }^{11-13)}$

In this study, we aimed to investigate the dynamics of the early vascular response to BP-SES using OCT to better understand the mechanisms underlying its favorable outcomes.

\section{Methods}

Study design and population: We conducted a singlecenter prospective observational pilot study. Consecutive patients with STEMI who underwent PCI between June 2018 and November 2019 at our institution were eligible. We applied the following exclusion criteria: cardiogenic shock, left main disease at the culprit lesion, reference vessel diameter $<2.0$ or $\geq 4.5 \mathrm{~mm}$, chronic kidney disease defined as a serum creatinine $>2.0 \mathrm{mg} / \mathrm{dL}$, maintenance dialysis, life expectancy $<2$ years, planned surgery within 3 months, adverse reactions to aspirin or clopidogrel previously, warfarin intake before STEMI onset, age $<20$ years, pregnancy, and a previously treated culprit lesion in the stented segment. We further excluded patients who did not undergo OCT, did not show plaque rupture on OCT, and received DES other than BP-SES. Among the remaining patients, those who presented for the two-week follow-up OCT (2W-OCT) were enrolled in the study. We underwent OCT at 2 weeks in proportion to the previous study. ${ }^{3)}$ Written informed consent was obtained for participation in this study before follow-up OCT.

OCT-guided PCI was defined as OCT being used at least before the intervention (pre-PCI) and at the final angiography after the intervention (post-PCI). A STEMI was defined according to the Third Universal Definition of Myocardial Infarction. ${ }^{14}$ ) The BP-SES (Orsiro ${ }^{\circledR}$, BIOTRONIK, Berlin, Germany) design required ultrathin
(60 $\mu \mathrm{m}$ for stent diameters $\leq 3.0 \mathrm{~mm}, 80 \mu \mathrm{m}$ for stent diameters $>3.0 \mathrm{~mm}$ ) struts. We administered a loading dose of aspirin (81-200 mg) before PCI and either clopidogrel $(300 \mathrm{mg})$ or prasugrel $(20 \mathrm{mg})$ (the standard loading dose in Japan) immediately after PCI. Thienopyridine was selected at the discretion of the attending physicians. We recommended the continuation of dual antiplatelet therapy (DAPT) with aspirin and thienopyridine for 12 months after PCI.

The study protocol was approved by the Ethical Committee of Showa University Hospital. All procedures of the study complied with the 1964 Declaration of Helsinki and its later amendments. All patients provided written informed consent before they were enrolled.

Coronary angiography and OCT image acquisition: Coronary angiography was obtained based on a standard series of multiple projections after the intracoronary injection of nitroglycerin. OCT was acquired pre- and postPCI, and 2 weeks ( $14 \pm 4$ days) after staged PCI for residual stenotic lesions or during follow-up angiography for evaluation before discharge. We used an OCT system (ILUMIEN $^{\mathrm{TM}}$ or ILUMIEN OPTIS) and an OCT catheter (Dragonfly $^{\mathrm{TM}} /$ Dragonfly JP/Dragonfly $^{\mathrm{TM}}$ OPTIS ${ }^{\mathrm{TM}}$ ) (all: Abbott Laboratories, Abbott Park, IL, USA).

All OCT images of the target lesion site (coronary segment from $5 \mathrm{~mm}$ distal to $5 \mathrm{~mm}$ proximal of the target lesion) were recorded with an automatic pullback system. We used a contrast agent to acquire OCT imaging, and lactated Ringer's solution or low-molecular-weight dextran were used according to the estimated glomerular filtration rate. The OCT images were digitally stored and submitted for offline analysis.

Image analysis and definitions and study endpoints: Offline OCT analysis was performed by two investigators, as previously reported, ${ }^{15)}$ who were blinded to the selection criteria of patients as well as the period since intervention (post-PCI or after 2 weeks). Quantitative OCT analysis was performed at every $1 \mathrm{~mm}$ interval, and the lumen area, stent area, inner lumen area (inner site of lumen or stent), and protrusion area of atherothrombotic burden (inner lumen area minus the stent area). The protrusion area was integrated into the protrusion volume $\left(\mathrm{mm}^{3}\right)$.

We defined a strut as uncovered if it was partially covered with tissue, and a strut as malapposed if the distance from the central reflection of the strut to the intimal surface of the arterial wall was $80 \mu \mathrm{m}$ or more for stent diameters $\leq 3.0 \mathrm{~mm}$, and $100 \mu \mathrm{m}$ or more for stent diameters $>3.0 \mathrm{~mm}$.

Qualitative OCT analyses were performed for each frame to diagnose the underlying mechanisms of the STEMI and in-stent structures. We used the following definitions according to previously published methods. ${ }^{16-19)}$ (1) plaque rupture: discontinuity of the fibrous cap with cavity formation; (2) plaque erosion: the presence of either a thrombus attached to an intact and visualized plaque, a luminal surface irregularity in the culprit lesion in the absence of a thrombus, or an attenuated underlying plaque without superficial lipid accumulation or calcification immediately proximal or distal to an attached thrombus; (3) calcified nodule: a fibrous cap disruption on a 


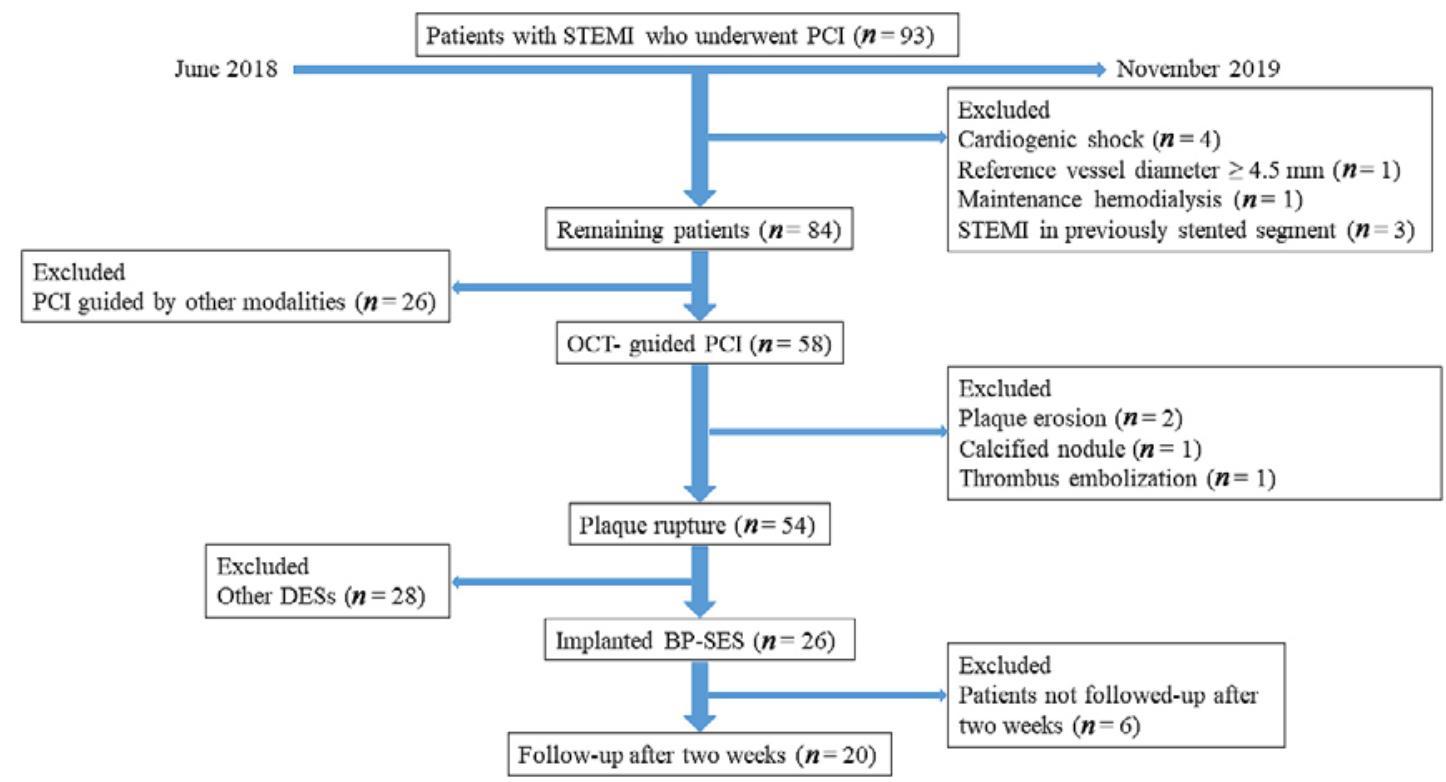

Figure 1. Study flow chart of patients with ST-segment elevation myocardial infarction treated with percutaneous coronary intervention. OCT was performed before the intervention and final angiography (post-PCI) and after two weeks. BP-SES indicates biodegradable polymers sirolimus-eluting stent; DES, drug-eluting stents; OCT, optical coherence tomography; PCI, percutaneous coronary intervention; and STEMI, ST-segment elevation myocardial infarction.

calcified plaque characterized by protruding calcification, superficial calcium, and the presence of substantive calcium proximal and/or distal to the lesion; (4) thrombus: a mass $\geq 250 \mu \mathrm{m}$ in height with an irregular surface attached to either the luminal surface or a stent strut, or floating within the lumen, causing signal attenuation; (5) smooth prolapse: a signal-rich tissue $\geq 250 \mu \mathrm{m}$ in height protruding between the struts without attenuation; (6) irregular protrusions: broad protrusion of material $\geq 250 \mu \mathrm{m}$ in height into the lumen between the stent struts with an irregular surface; (7) dissection: evidence of flaps $\geq 200$ $\mu \mathrm{m}$ in height within the stented segment and at the stent margin.

The primary endpoint was incomplete stent apposition measured as the percentage of malapposed struts. The secondary endpoints were the percentage of uncovered struts and the percentage of the reduction in atherothrombotic protrusion burden within the stent.

Statistical analysis: Qualitative data are presented as frequencies and quantitative data as the mean \pm standard deviation (SD). Categorical variables are expressed as numbers and percentages. We used the paired $t$-test and the Wilcoxon signed-rank and Fisher's exact tests to compare the post-PCI and $2 \mathrm{~W}$-OCT data, respectively. A $P$ value $<$ 0.05 was considered to denote a statistically significant difference.

We conducted all statistical analyses using $\mathrm{JMP}^{\circledR}$ Pro software (version 14; SAS Institute Inc., Cary, NC, USA).

\section{Results}

Baseline characteristics: Figure 1 shows the flow of patients through the study. Overall, 93 consecutive patients were eligible for participation. We initially excluded nine patients: four presented with cardiac shock, one with a reference vessel diameter of $\geq 4.5 \mathrm{~mm}$, one with maintenance hemodialysis, and three with stent thrombosis. Among the remaining patients, we excluded 26 patients who did not undergo OCT, four patients who did not show plaque rupture on OCT, and 28 patients who received DES other than BP-SES. Finally, we enrolled 20 patients who underwent $2 \mathrm{~W}-\mathrm{OCT}$.

Table I shows the baseline clinical, lesional, and procedural characteristics of the patients. BP-SES were placed after manual thrombus aspiration and balloon dilation in all cases. Only one patient $(5.0 \%)$ had received chronic antiplatelet therapy with aspirin before the onset of STEMI. All patients were started on prasugrel, a thienopyridine, after stent implantation, and DAPT was continued.

OCT findings: All 20 patients successfully underwent pre-PCI, post-PCI, and 2W-OCT. Figure 2 shows the representative cases of the three serial OCTs. In all patients, the pre-PCI showed the rupture site and thrombus. The post-PCI showed the situation after BP-SES implantation to the target lesion with plaque rupture and thrombi caused by malapposed struts, uncovered struts, and protrusion of atherothrombotic burden.

The 2W-OCT showed that the primary endpoint of incomplete stent apposition improved: the percentage of malapposed struts decreased from $4.7 \pm 3.3 \%$ post-PCI to $0.9 \pm 1.2 \%$ after 2 weeks $(P<0.0001)$.

Regarding the secondary endpoints, we found that the percentage of uncovered struts also significantly decreased from after the procedure to 2 weeks later $(69.8 \pm$ $18.3 \%$ post-PCI to $29.6 \pm 11.0 \%$ in the $2 \mathrm{~W}$-OCT, $P<$ $0.0001)$. The incidence of thrombi and irregular protrusion did not significantly differ between post-PCI and $2 \mathrm{~W}-\mathrm{OCT}$ 
Table I. Baseline Clinical, Lesional, and Procedural Characteristics

\begin{tabular}{|c|c|}
\hline Variable & \\
\hline Patients, $n$ & 20 \\
\hline Age, years & $62.7 \pm 11.3$ \\
\hline Sex, male, $n(\%)$ & $17(85)$ \\
\hline Height, cm & $164.6 \pm 11.7$ \\
\hline Body weight, kg & $66.6 \pm 13.0$ \\
\hline Diabetes, $n(\%)$ & $7(35)$ \\
\hline Hypertension, $n(\%)$ & $9(45)$ \\
\hline Dyslipidemia, $n(\%)$ & $11(55)$ \\
\hline $\mathrm{eGFR}<60, n(\%)$ & $3(15)$ \\
\hline Smoking, $n(\%)$ & $16(80)$ \\
\hline Family history, $n(\%)$ & $2(10)$ \\
\hline $\mathrm{LDL}-\mathrm{C}, \mathrm{mg} / \mathrm{dL}$ & $124.6 \pm 32.4$ \\
\hline HDL-C, mg/dL & $45.7 \pm 10.3$ \\
\hline non-HDL-C, mg/dL & $148.9 \pm 40.0$ \\
\hline $\mathrm{TG}, \mathrm{mg} / \mathrm{dL}$ & $164.5 \pm 164.9$ \\
\hline HbAlc, $\%$ & $6.2 \pm 1.0$ \\
\hline \multicolumn{2}{|l|}{ Culprit vessel, $n(\%)$} \\
\hline LAD, RCA, LCX & $6(30), 12(60), 2(10)$ \\
\hline \multicolumn{2}{|l|}{ TIMI-flow, $n(\%)$} \\
\hline $3,2,1,0$ & $0(0), 2(10), 1(5), 17(85)$ \\
\hline Bifurcation lesions, $n(\%)$ & $4(20)$ \\
\hline \multicolumn{2}{|l|}{ AHA/ACC lesion type, $n$} \\
\hline $\mathrm{A}, \mathrm{B} 1, \mathrm{~B} 2, \mathrm{C}$ & $0(0), 3(15), 13(65), 4(20)$ \\
\hline Door to balloon time, minutes & $69.4 \pm 54.4$ \\
\hline Used stent number, $n$ & $1.13 \pm 0.34$ \\
\hline Used stent diameter, $\mathrm{mm}$ & $3.25 \pm 0.35$ \\
\hline Used stent thickness, $\mu \mathrm{m}$ & $69 \pm 10.2$ \\
\hline Final maximum inflation pressure, atm & $15.8 \pm 5.2$ \\
\hline Aspiration catheter uses, $n(\%)$ & $20(100)$ \\
\hline Peak CPK, mg/dL & $3190(311-9071)$ \\
\hline Peak CPK-MB, mg/dL & $205(24-568)$ \\
\hline Hemoglobin, mg/dL & $14.5 \pm 2.3$ \\
\hline \multicolumn{2}{|l|}{ Chronic antiplatelet use prior to STEMI, $n(\%)$} \\
\hline Aspirin, clopidogrel, prasugrel & $1(5), 0(0), 0(0)$ \\
\hline Dual antiplatelet therapy at 2 weeks, $n(\%)$ & $20(100)$ \\
\hline \multicolumn{2}{|l|}{ Thienopyridine use, $n(\%)$} \\
\hline Clopidogrel, prasugrel & $0(0), 20(100)$ \\
\hline Anticoagulation drugs use, $n(\%)$ & $0(0)$ \\
\hline
\end{tabular}

$(P=0.5$ and 0.4 , respectively), but the maximum protrusion area of atherothrombotic burden was statistically significantly reduced $\left(1.36 \pm 0.70 \mathrm{~mm}^{2}\right.$ post-PCI to $0.98 \pm$ $0.55 \mathrm{~mm}^{2}$ at the $\left.2 \mathrm{~W}-\mathrm{OCT}, P=0.004\right)$. The protrusion volume of atherothrombotic burden decreased insignificantly $\left(15.4 \pm 11.4 \mathrm{~mm}^{3}\right.$ post-PCI to $12.2 \pm 8.5 \mathrm{~mm}^{3}$ at $2 \mathrm{~W}$ OCT, $P=0.4)$. The individual changes from the post-PCI to the $2 \mathrm{~W}-\mathrm{OCT}$ are plotted in Figure 3. Table II summarizes the other quantitative OCT variables.

\section{Discussion}

This prospective observational study of 20 patients with STEMI after plaque rupture was the first to assess the early vascular response to BP-SES implantation with serial OCT investigations before, immediately after, and 2 weeks after PCI. Two weeks after PCI, we found a (1) significant reduction in incomplete stent apposition, (2) rapid progression of strut coverage, and (3) significant re- duction of the intra-stent atherothrombotic burden compared with immediately after the procedure. The previous study $^{3)}$ showed early progression of strut coverage (63 \pm $20 \%$ post-PCI to $21 \pm 14 \%$ at $2 \mathrm{~W}$-OCT, $P<0.0001$ ), and improvements incomplete stent apposition $(7.3 \pm 9.0 \%$ post-PCI to $4.7 \pm 5.9 \%$ at $2 \mathrm{~W}$-OCT, $P=0.005)$. Our study showed a similar tendency, though we could not compare directly.

Pathological studies ${ }^{20,21)}$ have proposed the three most common mechanisms of coronary thrombosis causing ACS to be plaque rupture, plaque erosion, and calcified nodules. In patients with STEMI, plaque rupture is the most frequent underlying mechanism, whereas the other two mechanisms are more often seen in patients with nonSTEMI. ${ }^{17)}$ Patients with STEMI caused by plaque rupture show a poorer long-term prognosis compared with those who have other underlying mechanisms..$^{22,23)}$

Mechanisms of stent thrombosis assessed by intracoronary imaging: The absolute risk of stent thrombosis is 
A
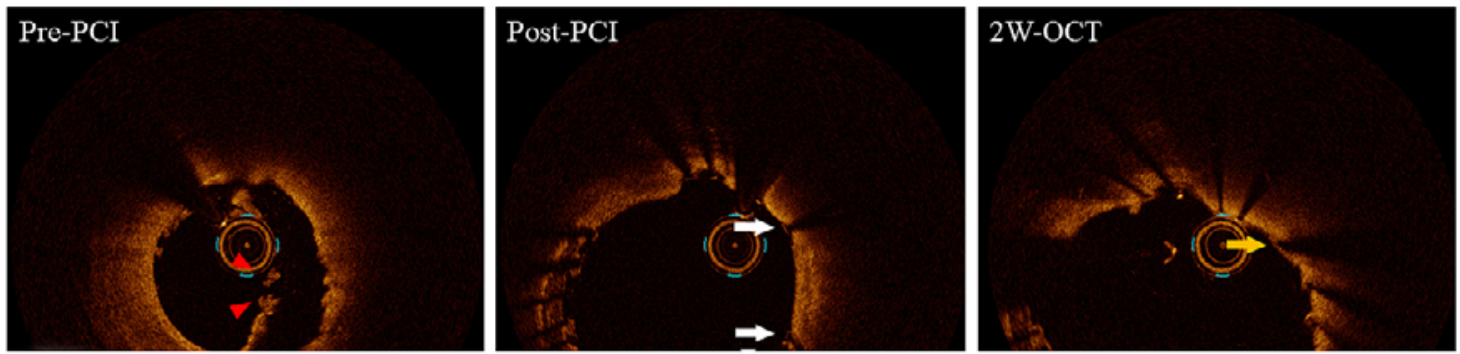

$\mathrm{B}$
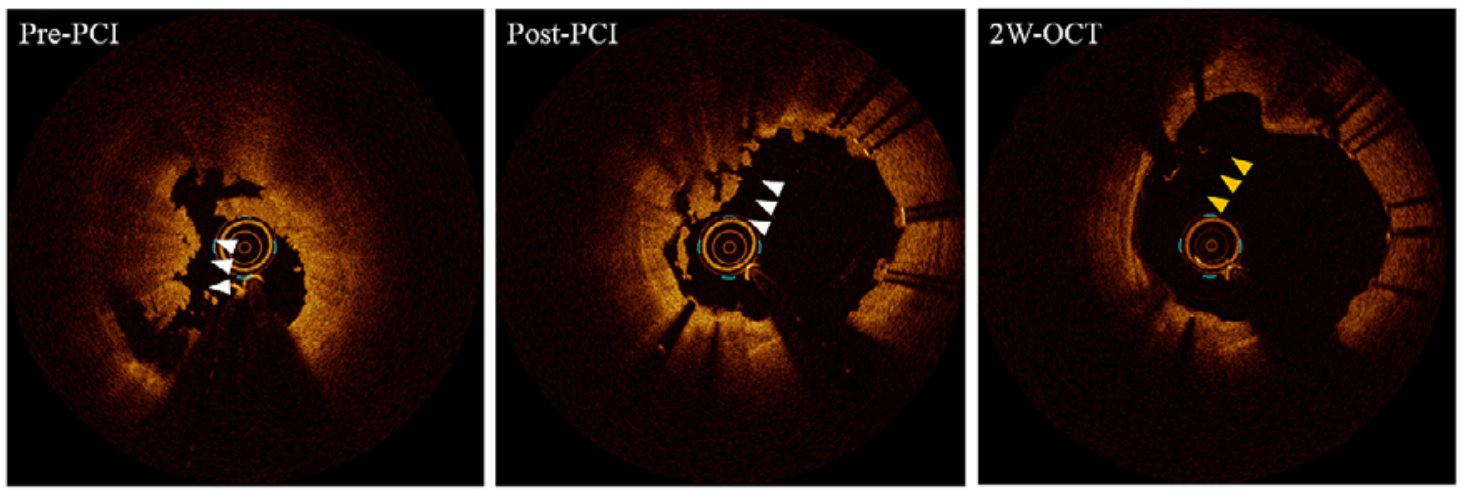

$\mathrm{C}$
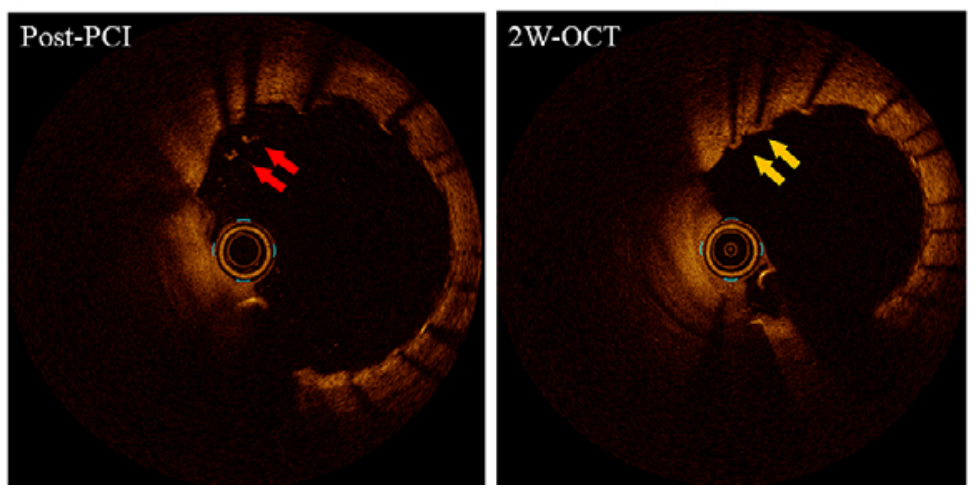

Figure 2. Representative serial optical coherence tomography images before, directly after, and 2 weeks after percutaneous coronary intervention. A: Female, 74 years old, STEMI of RCA. Red arrowheads indicate plaque rupture, white arrows indicate uncovered struts, white arrowheads indicate the protrusion of atherothrombotic burden, orange arrows indicate covered struts, orange arrowheads indicate the reduction of atherothrombotic burden. B: Male, 44 years old, STEMI of LAD. White arrowheads indicate the protrusion of atherothrombotic burden, orange arrowheads indicate the reduction of atherothrombotic burden. C: Female, 71 years old, STEMI of RCA. Red arrows indicate malapposed struts, orange arrows indicate covered struts. OCT indicates optical coherence tomography; PCI, percutaneous coronary intervention; $2 \mathrm{~W}$, two weeks; RCA, right coronary artery; and LAD, left anterior descending coronary artery.

low after treatment with current-generation DES, ${ }^{24)}$ but stent thrombosis is a residual problem of stent implantation after PCI. Various risk factors for stent thrombosis have been reported. ${ }^{25)}$ In terms of early stent thrombosis, procedural risk factors such as stent underexpansion, presence of residual dissection, impaired TIMI flow, and residual disease proximal or distal to the stent lesion are important predictors of stent thrombosis. ${ }^{26)}$ Adriaenssens, et $a l^{27)}$ reported malapposed and uncovered struts as the dominant findings identified by OCT in patients presenting with acute stent thrombosis. Moreover, tissue protrud- ing into the stent detected by intravascular ultrasound was reported as a contributing factor to early stent thrombo$\operatorname{sis}^{28)}$. In patients with STEMI, these findings are frequent. Our study showed a reduction in these risks during the very early phase after PCI, which could explain the favorable outcomes of BP-SES. ${ }^{6}$

Strut thickness and stent conformability of BP-SES: Thinner struts reduce stent-induced arterial injury and inflammation, facilitate faster endothelialization, and decrease thrombogenicity. ${ }^{2)}$ The significance of strut thickness has been studied since the era of BMS. In the ISAR- 

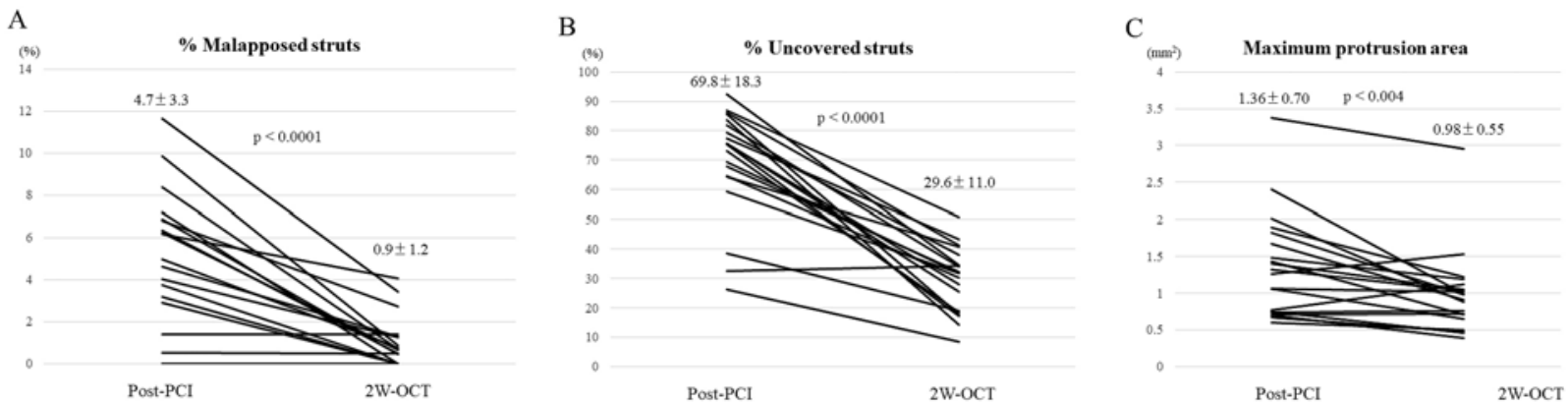

Figure 3. Primary and secondary endpoints of percutaneous coronary intervention in 20 patients with ST-segment elevation myocardial infarction assessed by OCT. A: Changes in the percentage of malapposed struts from directly after to two weeks after the intervention. B: Changes in the percentage of uncovered struts from directly after to 2 weeks after the intervention. C: Changes in the maximum protrusion area of the atherothrombotic burden from directly after to 2 weeks after the intervention. OCT indicates optical coherence tomography; and PCI, percutaneous coronary intervention.

Table II. Overall OCT Assessments at Post-Procedure and at 2 Weeks Follow-Up $(n=20)$

\begin{tabular}{lccc}
\hline Variables & Post-PCI & 2W-OCT & $P$ value \\
\hline Visual assessments & & & \\
No. of cross-section, $n$ & $24.1 \pm 6.1$ & $24.2 \pm 6.0$ & 0.9 \\
No. of struts, $n$ & $251.4 \pm 76.6$ & $253.2 \pm 58.4$ & 0.6 \\
No. of uncovered struts, $n$ & $176.5 \pm 72.9$ & $74.6 \pm 33.1$ & $<0.0001$ \\
\% uncovered struts, $\%$ & $69.8 \pm 18.3$ & $29.6 \pm 11.0$ & $<0.0001$ \\
No of malapposed struts, $n$ & $11.5 \pm 8.3$ & $2.1 \pm 2.4$ & 0.0003 \\
\% malapposed struts, $\%$ & $4.7 \pm 3.3$ & $0.9 \pm 1.2$ & $<0.0001$ \\
Quantitative measurements & & & \\
Average stent length, mm & $25.9 \pm 6.5$ & $25.6 \pm 7.2$ & 0.7 \\
Average stent area, mm ${ }^{2}$ & $8.54 \pm 1.86$ & $8.66 \pm 1.67$ & 0.9 \\
Minimum stent area, mm ${ }^{2}$ & $7.16 \pm 1.69$ & $7.21 \pm 1.61$ & 0.99 \\
Maximum stent area, mm ${ }^{2}$ & $10.36 \pm 2.35$ & $10.38 \pm 2.13$ & 0.96 \\
Average stent diameter, mm & $3.26 \pm 0.37$ & $3.29 \pm 0.33$ & 0.9 \\
Average lumen area, mm ${ }^{2}$ & $8.24 \pm 1.71$ & $8.48 \pm 1.63$ & 0.7 \\
Minimum lumen area, mm ${ }^{2}$ & $6.62 \pm 1.48$ & $6.83 \pm 1.57$ & 0.8 \\
Maximum lumen area, mm ${ }^{2}$ & $10.60 \pm 2.17$ & $10.35 \pm 2.03$ & 0.8 \\
Average lumen diameter, mm & $3.21 \pm 0.34$ & $3.25 \pm 0.32$ & 0.7 \\
Average protrusion area, mm ${ }^{2}$ & $0.53 \pm 0.37$ & $0.41 \pm 0.22$ & 0.4 \\
Minimum protrusion area, mm ${ }^{2}$ & $0.10 \pm 0.15$ & $0.10 \pm 0.06$ & 0.0051 \\
Maximum protrusion area, mm ${ }^{2}$ & $1.36 \pm 0.70$ & $0.98 \pm 0.55$ & 0.004 \\
Protrusion volume, mm ${ }^{3}$ & $15.39 \pm 11.40$ & $12.22 \pm 8.45$ & 0.4 \\
Qualitative OCT analysis & & & \\
Presence of thrombus, $n(\%)$ & $20(100)$ & $18(90)$ & 0.5 \\
Presence of irregular protrusion, $n(\%)$ & $18(90)$ & $15(75)$ & 0.4 \\
Presence of smooth protrusion, $n(\%)$ & $1(5)$ & $1(5)$ & 1 \\
Presence of dissection, $n(\%)$ & $2(10)$ & $0(0)$ & 0.5 \\
\hline PCI indicates percutaneous coronary intervention and OCT, optical coherence tomography.
\end{tabular}

STEREO study, ${ }^{29)}$ the use of thinner struts $(50 \mu \mathrm{m}$ compared with $140 \mu \mathrm{m}$ ) was associated with a significant reduction of angiographic and clinical restenosis after coronary artery stenting. Kolandaivelu, et $a l .{ }^{2}{ }^{2}$ reported that DES showed lower thrombogenicity than BMS in ex vivo models. In clinical settings, stent thrombosis was less frequent in DP-EES $(81 \mu \mathrm{m})$ than BMS, ${ }^{1)}$ and during the very early phase, DP-EES showed earlier progression of strut coverage and a reduction of thrombus area and length. ${ }^{3)}$ In a comparison between DES with the same design but with different strut thicknesses, thinner struts (81 $\mu \mathrm{m}$ as opposed to 140 and $132 \mu \mathrm{m})$ showed a lower incidence of uncovered struts and malapposed struts. ${ }^{30)}$
Recently, a meta-analysis of randomized trials showed that DES with ultrathin struts $(60-65 \mu \mathrm{m})$ were associated with a lower risk of target lesion failure and myocardial infarction compared with thin-strut DES. ${ }^{31)}$ Similarly, our study showed high antithrombogenicity and rapid progression of strut coverage using BP-SES.

On the other hand, we found a low percentage of malapposed struts and uncovered struts directly postprocedure and after 2 weeks. The low percentage of uncovered struts might be a consequence of the conformability of BP-SES. Generally, stents with thinner struts have more conformability, ${ }^{32}$ which might be associated with strut embedment in the vessel wall after stent placement. 
Strut width could influence the embedment of the strut because a device with a smaller contact area would generate a higher pressure on the vessel wall than one with a larger contact area if the same force was applied. ${ }^{33)}$ Both the width and depth of each strut of the BP-SES are 60 $\mu \mathrm{m}$, such that the cross-section of the stent strut is square. This advantageous feature of the stent strut might be responsible for the low percentage of uncovered struts.

Stent coating: Another contributing factor to the antithrombogenicity of BP-SES that we observed in our study might be chemical effects. It is known that thrombogenicity is determined by the electron charge of a stent. $^{34)}$ Hansi, et $a l .^{8)}$ investigated platelet adhesion, thrombus activation, and electron transfer of a stent coated with amorphous hydrogenated silicon carbide. They reported a markedly lower rate of platelet adhesion, fibrin activation, and electron transfer out of the stent compared with other stent coatings. This technology, called nanocoating used in BP-SES may play a favorable antithrombogenic role at 2 weeks when biodegradable polymer on top of nanocoating is still under ongoing degradation.

Furthermore, the polymer coating might be associated with antithrombogenicity. DP-EES coated with fluorinated polymer showed less acute thrombogenicity than DES coated with an abluminal biodegradable polymer, but no difference compared with BP-SES coated with a circumferential biodegradable polymer. ${ }^{9)}$ The circumferential coating of BP-SES might have an advantage over current DES using abluminal coating in thrombus-rich environments, in which protrusion is often formed inside of stents.

Clinical implications: Continuing DAPT beyond one year after DES placement has been recommended, ${ }^{35)}$ however, not all patients can benefit from this strategy because of the risk of bleeding. The optimal duration of DAPT has been repeatedly re-evaluated, and the safety of shortduration (1 to 3 months) DAPT has recently been demonstrated in several clinical trials. ${ }^{36-38)}$ In the STOPDAPT-2 trial, ${ }^{38)}$ subgroup analyses showed benefits of 1 month DAPT in terms of cardiovascular and bleeding events in patients with STEMI. The results of the present study support the safety of shorter DAPT duration after PCI in patients with STEMI.

Study limitations: This study has several limitations. First, this was an observational study with results based on data from a single center and small sample. Second, we did not compare the patients with a control group. Designing a head-to-head comparison between different drug-eluting stents would be ideal. Finally, patients were not evaluated by using other imaging modalities than OCT, such as intravascular ultrasound or angioscopy.

\section{Conclusion}

This study is the first to report very early vascular responses to BP-SES in patients with STEMI caused by plaque rupture. These responses entailed an early progression of strut coverage and resolution of atherothrombotic materials, showing a good safety profile of BP-SES during the very early phase after PCI.

\section{Acknowledgments}

The authors are deeply grateful to the following members of 1) Showa University: Katsuki Nagata and Noriko Iwaki, 2) Kobe Cardiovascular Core Laboratory and Micron Inc. Osaka, Japan: Hiromasa Otake, MD and Keiji Noutomi.

\section{Disclosure}

Conflicts of interest: None.

\section{References}

1. Sabate M, Cequier A, Iniguez A, et al. Everolimus-eluting stent versus bare-metal stent in ST-segment elevation myocardial infarction (EXAMINATION): 1 year results of a randomised controlled trial. Lancet 2012; 380: 1482-90.

2. Kolandaivelu K, Swaminathan R, Gibson WJ, et al. Stent thrombogenicity early in high-risk interventional settings is driven by stent design and deployment and protected by polymer-drug coatings. Circulation 2011; 123: 1400-9.

3. Morino Y, Terashita D, Otake H, et al. Early vascular responses to everolimus-eluting cobalt-chromium stent in the culprit lesions of st-elevation myocardial infarction: results from a multicenter prospective optical coherence tomography study (MECHANISM-AMI 2-week follow-up study). Cardiovasc Interv Ther 2019; 34: 14-24.

4. Kandzari DE, Mauri L, Koolen JJ, et al. Ultrathin, bioresorbable polymer sirolimus-eluting stents versus thin, durable polymer everolimus-eluting stents in patients undergoing coronary revascularisation (BIOFLOW V): a randomised trial. Lancet 2017; 390: $1843-52$.

5. Pilgrim T, Heg D, Roffi M, et al. Ultrathin strut biodegradable polymer sirolimus-eluting stent versus durable polymer everolimus-eluting stent for percutaneous coronary revascularisation (BIOSCIENCE): a randomised, single-blind, noninferiority trial. Lancet 2014; 384: 2111-22.

6. Iglesias JF, Muller O, Heg D, et al. Biodegradable polymer sirolimus-eluting stents versus durable polymer everolimuseluting stents in patients with ST-segment elevation myocardial infarction (BIOSTEMI): a single-blind, prospective, randomised superiority trial. Lancet 2019; 394: 1243-53.

7. Amabile N, Hammas S, Fradi S, et al. Intra-coronary thrombus evolution during acute coronary syndrome: regression assessment by serial optical coherence tomography analyses. Eur Heart J Cardiovasc Imaging 2015; 16: 433-40.

8. Hansi C, Arab A, Rzany A, Ahrens I, Bode C, Hehrlein C. Differences of platelet adhesion and thrombus activation on amorphous silicon carbide, magnesium alloy, stainless steel, and cobalt chromium stent surfaces. Catheter Cardiovasc Interv 2009; 73: 488-96.

9. Otsuka F, Cheng Q, Yahagi K, et al. Acute thrombogenicity of a durable polymer everolimus-eluting stent relative to contemporary drug-eluting stents with biodegradable polymer coatings assessed ex vivo in a swine shunt model. JACC Cardiovasc Interv 2015; 8: 1248-60.

10. Nakamura M, Yamagishi M, Ueno T, et al. Current antiplatelet therapy for Japanese patients with ST elevation acute myocardial infarction: J-AMI registry. Cardiovasc Interv Ther 2013; 28 : 162-9.

11. Matsumoto D, Shite J, Shinke T, et al. Neointimal coverage of sirolimus-eluting stents at 6-month follow-up: evaluated by optical coherence tomography. Eur Heart J 2007; 28: 961-7.

12. Ino $\mathrm{Y}$, Kubo $\mathrm{T}$, Tanaka A, et al. Comparison of vascular response between everolimus-eluting stent and bare metal stent implantation in ST-segment elevation myocardial infarction as- 
sessed by optical coherence tomography. Eur Heart J Cardiovasc Imaging 2015; 16: 513-20.

13. Sanuki Y, Sonoda S, Muraoka Y, et al. Contribution of poststent irregular protrusion to subsequent in-stent neoatherosclerosis after the second-generation drug-eluting stent implantation. Int Heart J 2018; 59: 307-14.

14. Thygesen K, Alpert JS, Jaffe AS, et al. Third universal definition of myocardial infarction. Circulation 2012; 126: 2020-35.

15. Karjalainen PP, Varho V, Nammas W, et al. Early neointimal coverage and vasodilator response following biodegradable polymer sirolimus-eluting vs. durable polymer zotarolimuseluting stents in patients with acute coronary syndromeHATTRICK-OCT trial. Circ J 2015; 79: 360-7.

16. Tearney GJ, Regar E, Akasaka T, et al. Consensus standards for acquisition, measurement, and reporting of intravascular optical coherence tomography studies: a report from the International Working Group for Intravascular Optical Coherence Tomography Standardization and Validation. J Am Coll Cardiol 2012; 59: 1058-72.

17. Jia $\mathrm{H}$, Abtahian $\mathrm{F}$, Aguirre $\mathrm{AD}$, et al. In vivo diagnosis of plaque erosion and calcified nodule in patients with acute coronary syndrome by intravascular optical coherence tomography. J Am Coll Cardiol 2013; 62: 1748-58.

18. Shinke T, Itoh T, Ishida M, et al. Early and mid-term vascular responses to optical coherence tomography-guided everolimuseluting stent implantation in stable coronary artery disease. Can J Cardiol 2019; 35: 1513-22.

19. Soeda T, Uemura S, Park SJ, et al. Incidence and clinical significance of poststent optical coherence tomography findings: one-year follow-up study from a multicenter registry. Circulation 2015; 132: 1020-9.

20. Virmani R, Kolodgie FD, Burke AP, Farb A, Schwartz SM. Lessons from sudden coronary death: a comprehensive morphological classification scheme for atherosclerotic lesions. Arterioscler Thromb Vascular Biol 2000; 20: 1262-75.

21. Naghavi M, Libby P, Falk E, et al. From vulnerable plaque to vulnerable patient: a call for new definitions and risk assessment strategies: Part I. Circulation 2003; 108: 1664-72.

22. Yonetsu T, Lee T, Murai T, et al. Plaque morphologies and the clinical prognosis of acute coronary syndrome caused by lesions with intact fibrous cap diagnosed by optical coherence tomography. Int J Cardiol 2016; 203: 766-74.

23. Niccoli G, Montone RA, Di Vito L, et al. Plaque rupture and intact fibrous cap assessed by optical coherence tomography portend different outcomes in patients with acute coronary syndrome. Eur Heart J 2015; 36: 1377-84.

24. Byrne RA, Serruys PW, Baumbach A, et al. Report of a European society of cardiology-European Association of Percutaneous Cardiovascular Interventions Task Force on the evaluation of coronary stents in Europe: executive summary. Eur Heart J 2015; 36: 2608-20.

25. Byrne RA, Joner M, Kastrati A. Stent thrombosis and restenosis: what have we learned and where are we going? The Andreas
Grüntzig Lecture ESC 2014. Eur Heart J 2015; 36: 3320-31.

26. van Werkum JW, Heestermans AA, Zomer AC, et al. Predictors of coronary stent thrombosis: the Dutch Stent Thrombosis Registry. J Am Coll Cardiol 2009; 53: 1399-409.

27. Adriaenssens T, Joner M, Godschalk TC, et al. Optical coherence tomography findings in patients with coronary stent thrombosis: a report of the PRESTIGE Consortium (Prevention of Late Stent Thrombosis by an Interdisciplinary Global European Effort). Circulation 2017; 136: 1007-21.

28. Choi SY, Witzenbichler B, Maehara A, et al. Intravascular ultrasound findings of early stent thrombosis after primary percutaneous intervention in acute myocardial infarction: a Harmonizing Outcomes with Revascularization and Stents in Acute Myocardial Infarction (HORIZONS-AMI) substudy. Circ Cardiovasc Interv 2011; 4: 239-47.

29. Kastrati A, Mehilli J, Dirschinger J, et al. Intracoronary stenting and angiographic results: strut thickness effect on restenosis outcome (ISAR-STEREO) trial. Circulation 2001; 103: 281621 .

30. Toledano Delgado FJ, Alvarez-Ossorio MP, de Lezo Cruz-Conde JS, et al. Optical coherence tomography evaluation of late strut coverage patterns between first-generation drug-eluting stents and everolimus-eluting stent. Catheter Cardiovasc Interv 2014; 84: 720-6.

31. Bangalore S, Toklu B, Patel N, et al. Newer-generation ultrathin strut drug-eluting stents versus older second-generation thicker strut drug-eluting stents for coronary artery disease. Circulation 2018; 138: 2216-26.

32. Colombo A, Stankovic G, Moses JW. Selection of coronary stents. J Am Coll Cardiol 2002; 40: 1021-33.

33. Serruys PW, Suwannasom P, Nakatani S, Onuma Y. Snowshoe versus ice skate for scaffolding of disrupted vessel wall. JACC Cardiovasc Interv 2015; 8: 910-3.

34. Hehrlein C, Zimmermann M, Metz J, Kübler W. Influence of surface texture and charge on the biocompatibility of endovascular stents. Coron Artery Dis 1995; 6: 581-6.

35. Mauri L, Kereiakes DJ, Yeh RW, et al. Twelve or 30 months of dual antiplatelet therapy after drug-eluting stents. N Eng J Med 2014; 371: 2155-66.

36. Mehran R, Baber U, Sharma SK, et al. Ticagrelor with or without Aspirin in High-Risk Patients after PCI. N Eng J Med 2019; 381: 2032-42.

37. Hahn JY, Song YB, Oh JH, et al. Effect of P2Y12 inhibitor monotherapy vs dual antiplatelet therapy on cardiovascular events in patients undergoing percutaneous coronary intervention: The SMART-CHOICE randomized clinical trial. JAMA 2019; 321: 2428-37.

38. Watanabe H, Domei T, Morimoto T, et al. Effect of 1-month dual antiplatelet therapy followed by clopidogrel vs 12 -month dual antiplatelet therapy on cardiovascular and bleeding events in patients receiving PCI: the STOPDAPT-2 randomized clinical trial. JAMA 2019; 321: 2414-27. 